\title{
Adaptive Fault-Tolerant Tracking Control of Nonaffine Nonlinear Systems with Actuator Failure
}

\author{
Hongcheng Zhou, ${ }^{1,2}$ Dezhi Xu, ${ }^{3}$ Daobo Wang, ${ }^{1}$ and Le Ge ${ }^{4}$ \\ ${ }^{1}$ College of Automation Engineering, Nanjing University of Aeronautics and Astronautics, Nanjing 210016, China \\ ${ }^{2}$ Institute of Information, Jinling Institute of Technology, Nanjing 211169, China \\ ${ }^{3}$ School of Automation, Nanjing Institute of Technology, Nanjing 211167, China \\ ${ }^{4}$ School of Electric Power Engineering, Nanjing Institute of Technology, Nanjing 211167, China
}

Correspondence should be addressed to Dezhi Xu; lutxdz@126.com

Received 16 February 2014; Accepted 26 June 2014; Published 16 October 2014

Academic Editor: Peng Shi

Copyright (C) 2014 Hongcheng Zhou et al. This is an open access article distributed under the Creative Commons Attribution License, which permits unrestricted use, distribution, and reproduction in any medium, provided the original work is properly cited.

This paper proposes an adaptive fault-tolerant control scheme for nonaffine nonlinear systems. A model approximation method which is a solution that bridges the gap between affine and nonaffine control systems is developed firstly. A joint estimation approach is based on unscented Kalman filter, in which both failure parameters and states are simultaneously estimated by means of the argument state vector composed of the unknown faults and states. Then, stability analysis is given for the closed-loop system. Finally, the proposed approach is verified using a three-degree-of-freedom simulation of a typical fighter aircraft and the significantly improved system response demonstrates the practical potential of the theoretic results obtained.

\section{Introduction}

The increasing demands on the performance of many modern systems will correspondingly increase the possibility of system failures. Faults may occur in any locations and dramatically change the system behaviour resulting in degradation or even instability. To improve system reliability and stability, fault-tolerant control (FTC) for dynamic systems has become an attractive topic and has received considerable attention during the past two decades. The FTC can be mainly classified into two types: passive and active [1]. In the passive approach, the same controller is used throughout the normal case as well as the fault case [2-5]. An active FTC system compensates for the effect of fault by synthesizing a new control strategy based on online accommodation [6-8]. Generally speaking, the active approach is less conservative than the passive one, which has increasingly been the main methodology in designing FTC systems [9].

On the other hand, tracking control plays an important role in the field of industrial production, aeronautics, and astronautics, such as a flexible robotic, aerospace vehicle.
Therefore, it has been a hot research topic for scientists and engineers over the past few years [10-12]. The tracking controller design of complex nonlinear systems is not easy work, particularly for nonaffine nonlinear systems. One nonlinear approach is the inverse system method. Although the existence of an inverse function can be guaranteed by the implicit function theorem [13], it is generally difficult to prescribe a technique to actually obtain such an inverse. In another approach, an integrator, that is, $u=\int_{o}^{t} \dot{u} \mathrm{dt}$, is introduced to a new control input $\dot{u}$. However, the relative degree of the augmented system is higher than that of the original system $[14,15]$.

As an important research direction of active FTC, fault accommodation (FA) is paid attention by many researchers. Recently, some results for fault estimation and accommodation have been obtained [16-18]. Compared with fault detection and isolation (FDI) only, fault estimation and accommodation of nonaffine nonlinear systems are not an easy task. About two parts of FA must be developed, such as a reconfigurable nonlinear tracking controller and a fault estimation (state unmeasured) module. As far as we know, most 
articles of fault-tolerant tracking control are mainly focused on affine systems and how to design tracking controller of nonaffine nonlinear systems, which is the main obstacle for fault-tolerant tracking control of nonaffine systems.

This paper addresses the fault-tolerant tracking control problem for nonaffine nonlinear systems in the presence of actuator faults. A novel dynamic model approximation method is first proposed to approximate the nonaffine nonlinear dynamics, which is a solution that bridges the gap between affine and nonaffine control systems. Then, the unscented Kalman filter (UKF) algorithm is employed to estimate plant states and faults from the measurable output. Recent studies on nonlinear systems [19] have shown improved execution of the UKF relative to the more usually used extended Kalman filter. Moreover, amendments to the base UKF algorithm have been shown to be competent of handling multimodal likelihood density functions and so forth, making it a very attractive and computationally inexpensive state estimation alternative to moving horizon estimator based methodologies [19]. Appropriate closed-loop system stability analysis is given by using the proposed adaptive fault-tolerant tracking control structure with the UKF.

The rest of this paper is organized as follows. Section 2 includes system description and problem formulation. The ideal FTC is given in Section 3. In Section 4, further, UKFbased FTC of nonaffine nonlinear systems is proposed, and appropriate closed-loop system stability analysis is given. Finally, the proposed approach is tested using a three-degreeof-freedom (3-DOF) unmanned aerial vehicle UAV point mass model.

\section{Problem Formulation}

Consider the following nonaffine nonlinear system:

$$
\begin{gathered}
\dot{x}=F\left(x, u_{c}\right), \\
y=h(x),
\end{gathered}
$$

where $x \in \mathbb{R}^{r}$ is the state vector, $u_{c} \in \mathbb{R}^{m}$ is the efficacious input vector, and $y \in \mathbb{R}^{n}$ is the output vector, respectively. $F(\cdot)$ and $h(\cdot)$ are the nonlinear functions. Let $\Omega_{\chi} \in \mathbb{R}^{p}$ be compact set defined by $\Omega_{\chi} \triangleq\left\{x|| x \mid \leqslant b_{\chi}\right\}$, where $b_{x}>0$ is a positive constant. The following assumption is made for system (1).

Assumption 1. $F\left(x, u_{c}\right)$ is $C^{1}$ for all $\left(x, u_{c}\right) \in \Omega_{\chi} \times \mathbb{R}$ and is a smooth function with respect to control input $u_{c}$.

If the control channel exists $u_{c}=\mu u$, where $\mu \in[0,1]$ is loss of effectiveness (LOE) factor, $\mu=0$ denotes the total LOE, and $0<\epsilon \leqslant \mu \leqslant 1$ denotes the partial LOE. So the failure model of (1) can be expressed as the following general formula:

$$
\begin{gathered}
\dot{x}=F(x, u, \mu), \\
y=h(x),
\end{gathered}
$$

where $\mu(t)$ describe fault signal and can be constant or time varying. The existence of failure can lead to steady state offsets. A significant concern in the formulation of the fault accommodation algorithm is the requisite tracking action in the closed-loop. A proper fault and state model must be designed such that they can be estimated from the measurable output. Here, the plant dynamics and measurements are described by the discrete nonlinear model

$$
\begin{aligned}
{\left[\begin{array}{c}
x(k+1) \\
\mu(k+1)
\end{array}\right] } & =\left[\begin{array}{c}
F(x(k), u(k), \mu(k)) \\
\mu(k)
\end{array}\right]+\left[\begin{array}{c}
\nu(k) \\
\omega(k)
\end{array}\right], \\
y(k) & =h(x(k))+\xi(k) .
\end{aligned}
$$

The process noise $\nu(k) \in \mathbb{R}^{r}, \omega(k) \in \mathbb{R}^{m}$, and measurement noise $\xi(k) \in \mathbb{R}^{n}$ are assumed to be additive zero-mean Gaussian white-noise processes with covariance matrices $Q(k), S(k)$, and $R(k)$, respectively.

\section{Ideal Fault-Tolerant Controller Design}

3.1. A Model Approximation Method. The problem of controlling the plants characterized by models that are nonaffine in the control input vector is a thorny one, especially for the tracking control. So far, concentrated research has been conducted for the controller design only for affine nonlinear systems. So, this subsection focuses on how to directly apply their results to the nonaffine systems.

From Assumption 1, the Taylor expansion of the nonlinear function $F(x, u, \mu)$ with respect to $u(t)$ around the neighborhood $u_{n}(t)$ can result in

$$
F(x, u, \mu)=F\left(x, u_{n}, \mu\right)+\left.\frac{\partial F(x, u, \mu)}{\partial u}\right|_{u=u_{n}}\left(u-u_{n}\right)+O(\cdot)
$$

where

$$
O(\cdot)=\left.\sum_{i=2}^{\infty} \frac{\partial^{i} F(x, u, \mu)}{\partial u^{i}}\right|_{u=u_{n}}\left(u-u_{n}\right)^{i}
$$

If we let $g\left(x, u_{n}, \mu\right)=\left.(\partial F(x, u, \mu) / \partial u)\right|_{u=u_{n}}, f\left(x, u_{n}, d, \mu\right)=$ $F\left(x, u_{n}, \mu\right)-g\left(x, u_{n}, \mu\right) u_{n}+E(x) d$, so we can rewrite (2) as

$$
\begin{gathered}
\dot{x}=f\left(x, u_{n}, \mu\right)+g\left(x, u_{n}, \mu\right) u+O(\cdot), \\
y=h(x) .
\end{gathered}
$$

Assumption 2. There exists a known constant $g_{M}$ such that $\left\|g\left(x, u_{n}, \mu\right)\right\| \leqslant g_{M}$ for all $\left(x, u_{n}, \mu\right) \in \Omega_{\chi} \times R$.

Lemma 3. If $\partial F(w) / \partial w$ exists and is continuous on $U$, that is, $F$ is $C^{1}$, then $F$ is locally Lipschitz on $U$.

Proof. See [20].

Proposition 4. There exists a constant $L_{2}$ which satisfies the inequality $\|O(\cdot)\| \leqslant L_{2}\left\|u-u_{n}\right\|$ for all $x \in \Omega_{\chi}$. 
Proof. Rearranging (4) with respect to $O(\cdot)$, we obtain

$$
O(\cdot)=F(x, u, \mu)-F\left(x, u_{n}, \mu\right)-g\left(x, u_{n}, \mu\right)\left(u-u_{n}\right) \text {. }
$$

From Assumptions 1 and 2 and Lemma 3, there exist $L_{1}$ and $g_{M}$ such that

$$
\begin{gathered}
\left\|F(x, u, \mu)-F\left(x, u_{n}, \mu\right)\right\| \leqslant L_{1}\left\|u-u_{n}\right\|, \\
\left\|g\left(x, u_{n}, \mu\right)\right\|\left\|u-u_{n}\right\| \leqslant g_{M}\left\|u-u_{n}\right\| .
\end{gathered}
$$

Taking the absolute value on both sides of (7) and using (8), we can easily induce

$$
\begin{aligned}
\|O(\cdot)\| \leqslant & \left\|F(x, u, \mu)-F\left(x, u_{n}, \mu\right)\right\| \\
& +\left\|g\left(x, u_{n}, \mu\right)\right\|\left\|u-u_{n}\right\| \\
\leqslant & L_{1}\left\|u-u_{n}\right\|+g_{M}\left\|u-u_{n}\right\| \\
= & L_{2}\left\|u-u_{n}\right\|,
\end{aligned}
$$

where $L_{2}=L_{1}+g_{M}$.

From (9), it can be seen that if we let $\lim \left\|u-u_{n}\right\|=0$, then $\lim \|O(\cdot)\|=0$. In many actual process control systems and flight control systems, $\|u(t)-u(t-\tau)\| \in[0, \delta]$ is a physical restriction of many practical systems because their actuators cannot change too fast due to system "inertia." So in $[21,22]$, the $u(t-\tau)$ is used to replace $u_{n}$. However, if the timedelay $\tau$ is selected too large, the precision of approximation of simplified model will be reduced. So the selection of $\tau$ often requires experience. Theoretically, the smaller $\tau$ can provide the better precision of global approximation. If $\tau=0$, the best precision of global approximation can be achieved. But $u$ is control law to be solved, so it is unable to be realized. In order to obtain exact time-varying trim point $u_{n}$, here, further improvement of above proposed method is given as follows. Consider lag property of the filtering as

$$
\dot{u}_{n}=-\zeta u_{n}+\zeta u
$$

Then $\lim _{\zeta \rightarrow \infty} u_{n}=u$. So use filter (10); it can be ensured that $\lim _{\zeta \rightarrow \infty}\|O(\cdot)\|=0$.

Remark 5. Here, $\zeta \rightarrow \infty$ is only a rigorous expression for mathematics meanings; in general, $\zeta \in[5,50]$. Filter (10) is not unique. The filtering $u_{n}$ can be completely replaced by other filtering equation, such as higher-order differentiator [23].

From above analysis, system (2) can be described as an affine system with time-varying parameters by the following:

$$
\begin{gathered}
\dot{u}_{n}=-\zeta u_{n}+\zeta u, \\
\dot{x}=f\left(x, u_{n}, \mu\right)+g\left(x, u_{n}, \mu\right) u, \\
y=h(x) .
\end{gathered}
$$

3.2. Ideal Fault-Tolerant Controller. Let $r_{i}$, the linearizability index, be the minimum order of the derivative of $y_{i}(i=$ $1, \ldots, n)$ for which the coefficient of at least one $u_{k}(k=$ $1, \ldots, m)$ is not zero. When the Lie derivative notation is used, this derivative can be expressed as

$$
y_{i}^{\left(r_{i}\right)}=L_{f}^{r_{i}} h_{i}+\sum_{k=1}^{m} L_{g_{k}} L_{f}^{r_{i}-1} h_{i} \cdot u_{k}
$$

where the Lie derivatives are defined as

$$
\begin{gathered}
L_{f}^{0} h_{i}(x) \triangleq h_{i}(x), \\
L_{f}^{s} h_{i}(x)=\frac{\partial L_{f}^{s-1} h_{i}(x)}{\partial x} f\left(x, u_{n}, \mu\right), \\
L_{g_{k}} L_{f}^{s-1} h_{i}(x)=\frac{\partial L_{f}^{s-1} h_{i}(x)}{\partial x} g_{k}\left(x, u_{n}, \mu\right), \\
i=1,2, \ldots, n, \quad k=1,2, \ldots, m .
\end{gathered}
$$

Given that the nonlinear system is input-output (I/O) linearizable, for each output $y_{i}$ there exists a linearizability index $r_{i}$.

Assumption 6. The drift term, $L_{f}^{r_{i}} h_{i}$, and the control gain, $L_{g_{k}} L_{f}^{r_{i}-1} h_{i}$, of the I/O dynamics (12) are globally bounded and Lipschitz.

Define

$$
\begin{gathered}
\mathscr{F}(x)=\left[L_{f}^{r_{1}} h_{1}, \ldots, L_{f}^{r_{n}} h_{n}\right], \\
\mathscr{G}(x)=\left[\mathscr{G}_{1}(x), \ldots, \mathscr{G}_{m}(x)\right], \\
\text { with } \mathscr{G}_{l}(x)=\left[L_{g_{1}} L_{f}^{r_{i}-1} h_{i}, \ldots, L_{g_{m}} L_{f}^{r_{i}-1} h_{i}\right], \\
\mathscr{A}=\operatorname{diag}\left[A_{1}, \ldots, A_{n}\right], \\
\mathscr{B}=\operatorname{diag}\left[B_{1}, \ldots, B_{n}\right], \\
A_{i}=\left[\begin{array}{cccc}
0 & 1 & \cdots & 0 \\
\vdots & \vdots & \ldots & \vdots \\
0 & 0 & 0 & 1 \\
0 & 0 & 0 & 0
\end{array}\right]_{r_{i} \times r_{i}} \quad B_{i}=\left[\begin{array}{c}
0 \\
\vdots \\
0 \\
1
\end{array}\right]_{r_{i} \times 1} \quad C_{i}=\left[\begin{array}{c}
1 \\
\vdots \\
0 \\
0
\end{array}\right]_{1 \times r_{i}}
\end{gathered} .
$$

Define $\mathbb{x}=\left[y_{1}, \ldots, y_{1}^{\left(r_{1}-1\right)}, \ldots, y_{n}, \ldots, y_{n}^{\left(r_{n}-1\right)}\right]^{T}$; then (12) can be rewritten as

$$
\begin{gathered}
\dot{\mathbb{x}}=\mathscr{A} \mathbb{x}+\mathscr{B}[\mathscr{F}(x)+\mathscr{G}(x) u], \\
y=\mathscr{C} \mathbb{} .
\end{gathered}
$$


For the given references $y_{1 m}, \ldots, y_{n m}$, define the tracking errors as

$$
\begin{gathered}
e_{1}=y_{1}-y_{1 m}, \\
\vdots \\
e_{n}=y_{n}-y_{n m}
\end{gathered}
$$

and $Y_{m}=\left[y_{1 m}, \ldots, y_{1 m}^{\left(r_{1}-1\right)}, \ldots, y_{n m}, \ldots, y_{n m}^{\left(r_{n}-1\right)}\right]^{T}$; then $\mathbb{e}=$ $\left[e_{1}, \ldots, e_{1}^{\left(r_{1}-1\right)}, \ldots, e_{n}, \ldots, e_{n}^{\left(r_{n}-1\right)}\right]^{T}$.

So the control law can be chosen with (10) as

$$
\begin{gathered}
\dot{u}_{n}=-\zeta u_{n}+\zeta u, \\
u=\mathscr{G}^{-1}\left(x, u_{n}, \mu\right)\left[-\mathscr{F}\left(x, u_{n}, \mu\right)+y_{m}^{r}+\mathscr{K}_{c}^{T} \mathbb{}\right]
\end{gathered}
$$

where $y_{m}^{(r)}=\left[y_{1 m}^{\left(r_{1}\right)}, \ldots, y_{n m}^{\left(r_{n}\right)}\right]^{T}$. Substituting (17) into (15) yields

$$
\dot{\mathrm{e}}=\left(\mathscr{A}-\mathscr{B} \mathscr{K}_{c}^{T}\right) \mathbb{e} .
$$

It can be seen that we can design the gain matrix $\mathscr{K}_{c}$ by following Riccati equation:

$$
\left(\mathscr{A}-\mathscr{B} \mathscr{K}_{c}^{T}\right)^{T} \mathscr{H}+\mathscr{H}\left(\mathscr{A}-\mathscr{B} \mathscr{K}_{c}^{T}\right)=-Q
$$

where $Q=Q^{T}>0$. Hence, if $x, d$, and $\mu$ are known, the above controller achieves the control objective. However, since states and fault information are generally unknown, the adaptive fault-tolerant controller is implemented by replacing the failure-related parameters and their estimates, as discussed below.

\section{Adaptive Fault-Tolerant Controller Design}

4.1. Unscented Kalman Filter Based Fault-Tolerant Controller. Define the variables $\mathbf{x}=\left[x^{T}, \mu^{T}\right]^{T}$; the adaptive fault-tolerant controller is now chosen in the form

$$
u=\mathscr{G}^{-1}\left(\widehat{\mathbf{x}}, u_{n}\right)\left[-\mathscr{F}\left(\widehat{\mathbf{x}}, u_{n}\right)+y_{m}^{r}+\mathscr{K}_{c}^{T} \mathbb{e}\right] .
$$

Next, we recapitulate the UKF state estimation algorithm utilized in this study. The model (3) can be expressed equivalently by the discrete-time model

$$
\begin{aligned}
\mathbf{x}(k+1) & =\mathbf{f}(\mathbf{x}(k), u(k))+v(k), \\
y(k) & =\mathbf{h}(\mathbf{x}(k))+\xi(k),
\end{aligned}
$$

where $v=\left[v^{T}, \omega^{T}\right]^{T}$. An $n^{a}$-dimensional vector $\mathbf{x}^{a}(k-1$ $k-1)$ is then defined by augmenting the state vector with the process and measurement noise vectors following in

$$
\begin{aligned}
\mathbf{x}^{a} & (k-1 \mid k-1) \\
& =\left[\mathbf{x}^{T}(k-1 \mid k-1), v^{T}(k-1), \xi^{T}(k-1)\right]^{T} .
\end{aligned}
$$

Since the process and measurement noise are supposed to be zero-mean, the mean $\widehat{\mathbf{x}}^{a}(k-1 \mid k-1)$ of the augmented state vector is presented by

$$
\widehat{\mathbf{x}}^{a}(k-1 \mid k-1)=\left[\widehat{\mathbf{x}}^{T}(k-1 \mid k-1), \mathbf{0}^{1 \times(r+2 m)}, \mathbf{0}^{1 \times n}\right]^{T} .
$$

Thus, the discrete-time nonlinear model (21) can be rephrased in terms of the augmented vector yielding

$$
\begin{gathered}
\mathbf{x}^{a}(k+1 \mid k)=\mathbf{f}^{a}\left(\mathbf{x}^{a}(k \mid k), u(k)\right), \\
y(k)=\mathbf{h}^{a}\left(\mathbf{x}^{a}(k \mid k)\right) .
\end{gathered}
$$

The covariance matrix $P^{a}(k \mid k)$ of the augmented system can be calculated from

$$
P^{a}(k \mid k)=\left[\begin{array}{ccc}
\mathbf{P}(k \mid k) & \mathbf{0} & \mathbf{0} \\
\mathbf{0} & \mathbf{Q}(k) & P^{v \xi}(k) \\
\mathbf{0} & P^{\xi v}(k) & R(k)
\end{array}\right],
$$

where $\mathbf{P}(k \mid k)$ is the estimation error covariance of the state $\mathbf{x}(k), \mathbf{Q}(k)$ is the covariance of the process noise $v(k)$, and $R(k)$ is the covariance of the measurement noise $\xi(k)$. The prediction step of the UKF algorithm includes the propagation of a given set of sigma points within the nonlinear system to produce a consistent set of changed points which are then used to make predictions of the state estimates. Now, the set of $\left(2 n^{a}+1\right)$ sigma points $\chi_{i}(k-1 \mid k-1)$ is computed according to the algorithm

$$
\begin{aligned}
\chi_{0}^{a}(k-1 \mid k-1)=\widehat{\mathbf{x}}^{a}(k-1 \mid k-1), & \\
\chi_{i}^{a}(k-1 \mid k-1)= & \widehat{\mathbf{x}}^{a}(k-1 \mid k-1) \\
+ & {\left[\left(n^{a}+\kappa\right) P^{a}(k-1 \mid k-1)\right]_{i}^{1 / 2}, } \\
\chi_{i+n^{a}}^{a}(k-1 \mid k-1)= & \widehat{\mathbf{x}}^{a}(k-1 \mid k-1) \\
- & {\left[\left(n^{a}+\kappa\right) P^{a}(k-1 \mid k-1)\right]_{i}^{1 / 2} }
\end{aligned}
$$

for all integers $i \in\left[0,2 n^{a}\right]$. Here, $\kappa$ is a scalar parameter used to "fine tune" higher order moments of the supply in order to reduce global prediction errors. Julier et al. [24] recommend that it be chosen such that $n^{a}+\kappa=3$. In the monitoring, we represent the total sigma point set by $\chi^{a}(k-1 \mid k-1)$.

Each sigma point in the set $\chi^{a}(k-1 \mid k-1)$ is propagated via the nonlinear process model over the sampling interval $[t-1, t]$, in order to produce a set of changed points $\chi_{i}(k-1 \mid$ $k-1)$ given by

$$
\chi_{i}(k \mid k-1)=\mathbf{f}^{a}\left(\chi_{i}^{a}(k-1 \mid k-1), u(k-1)\right) .
$$

The predicted state estimate $\widehat{\mathbf{x}}(k \mid k-1)$ is computed as a weighted average of the changed points given by

$$
\widehat{\mathbf{x}}(k \mid k-1)=\sum_{i=0}^{2 n^{a}} W_{i} \chi_{i}(k \mid k-1),
$$


where the weighting factors $W_{i}$ are selected corresponding to the algorithm

$$
W_{i}= \begin{cases}\frac{\kappa}{\left(n^{a}+\kappa\right)}, & \text { if } i=0 \\ \frac{1}{2\left(n^{a}+\kappa\right)}, & \text { if } i \neq 0 .\end{cases}
$$

The predicted estimation error covariance $\mathbf{P}(k \mid k-1)$ is computed from the weighted outer result of the changed points given by

$$
\begin{aligned}
\mathbf{P}(k \mid k-1)=\sum_{i=0}^{2 n^{a}} W_{i} & {\left[\chi_{i}(k \mid k-1)-\widehat{\mathbf{x}}(k \mid k-1)\right] } \\
& \times\left[\chi_{i}(k \mid k-1)-\widehat{\mathbf{x}}(k \mid k-1)\right]^{T} .
\end{aligned}
$$

The propagated set of sigma points $\chi(k \mid k-1)$ are then represented within the nonlinear measurement function $\mathbf{h}^{a}(\cdot)$, generating a set of outputs $Y_{i}(k)$ communicated by

$$
Y_{i}(k)=\mathbf{h}^{a}(\chi(k \mid k-1)) .
$$

In a fashion analogous to the predicted state estimate, the predicted output $\widehat{y}(k)$ is computed as a weighted average of the represented outputs (31) given by

$$
\widehat{y}(k)=\sum_{i=0}^{2 n^{a}} W_{i} Y_{i}(k) .
$$

The novelty covariance $P_{y y}$ and the cross relationship $P_{x y}$ are calculated, respectively, from the following illustration:

$$
\begin{gathered}
P_{y y}=\sum_{i=0}^{2 n^{a}} W_{i}\left[Y_{i}(k)-\widehat{y}(k)\right]\left[Y_{i}(k)-\widehat{y}(k)\right]^{T}, \\
P_{x y}=\sum_{i=0}^{2 n^{a}} W_{i}\left[\chi_{i}(k \mid k-1)-\widehat{\mathbf{x}}(k \mid k-1)\right]\left[Y_{i}(k)-\widehat{y}(k)\right]^{T} .
\end{gathered}
$$

The measurement improvement terms for the unscented filter are presented by

$$
\begin{gathered}
\widehat{\mathbf{x}}(k \mid k)=\widehat{\mathbf{x}}(k \mid k-1)+K(k)(y(k)-\widehat{y}(k)), \\
\mathbf{P}(k \mid k)=\mathbf{P}(k \mid k-1)-K(k) P_{y y} K(k)^{T},
\end{gathered}
$$

where the Kalman gain $K(k)$ is computed from $K(k)=$ $P_{x y} P_{y y}^{-1}$.

4.2. Stability Analysis. Define estimation error $\widetilde{\mathbf{x}}=\widehat{\mathbf{x}}-\mathbf{x}$. Equation (15) can be equivalent as follows:

$$
\begin{gathered}
\dot{\mathbb{x}}=\mathscr{A} \mathbb{x}+\mathscr{B}\left[\mathscr{F}\left(\widehat{\mathbf{x}}, u_{n}\right)+\mathscr{G}\left(\widehat{\mathbf{x}}, u_{n}\right) u\right]+\mathscr{B} \mathscr{P} \widetilde{\mathbf{x}} \\
y=\mathscr{C} \mathbb{},
\end{gathered}
$$

where $\mathscr{P}=\partial \mathscr{F}\left(\mathbf{x}, u_{n}\right) /\left.\partial \mathbf{x}\right|_{\mathbf{x}=\phi}+\partial \mathscr{G}\left(\mathbf{x}, u_{n}\right) /\left.\partial \mathbf{x}\right|_{\mathbf{x}=\phi}$ with $\phi \epsilon$ $[\mathbf{x}, \widehat{\mathbf{x}}]$.
Substituting (20) into (35), the dynamics of close-loop can be obtained as

$$
\dot{\mathbb{e}}=\left(\mathscr{A}-\mathscr{B} \mathscr{K}_{c}^{T}\right) \mathbb{e}+\mathscr{B} \mathscr{P} \widetilde{\mathbf{x}} .
$$

Choose the following Lyapunov function:

$$
V=\mathbb{e}^{T} \mathscr{H} \mathbb{e} .
$$

The time derivative of $V$ is given by

$$
\dot{V}=-e^{T} Q \mathbb{Q}+2 e^{T} \mathscr{H} \mathscr{B} \mathscr{P} \widetilde{\mathbf{x}} .
$$

Under Young's inequality $2 a^{T} b \leqslant \varepsilon a^{T} a+\varepsilon^{-1} b^{T} b$, we have

$$
\begin{aligned}
& \dot{V} \leqslant-\lambda_{\min }(\mathscr{Q}) \Theta^{T} \mathscr{H} 巴+2 e^{T} \mathscr{H} \mathscr{B} \mathscr{P} \widetilde{\mathbf{x}} \\
& \leqslant-\frac{\lambda_{\min }(\mathscr{Q})}{\lambda_{\max }(\mathscr{H})} \Theta^{T} \mathscr{H} 巴+\mu_{1} \Theta^{T} \mathscr{H} 巴+\mu_{1}^{-1} \widetilde{\mathbf{x}}^{T} \mathscr{P} \mathscr{B}^{T} \mathscr{H} \mathscr{B} \mathscr{P} \widetilde{\mathbf{x}} \\
& \leqslant-\left[\frac{\lambda_{\min }(\mathscr{Q})}{\lambda_{\max }(\mathscr{H})}-\mu_{1}\right] V+\mu_{1}^{-1} \lambda_{\max }\left(\mathscr{P} \mathscr{B}^{T} \mathscr{H} \mathscr{B} \mathscr{P}\right)\|\widetilde{\mathbf{x}}\|^{2} \\
& \leqslant-\lambda V+\varphi(t)
\end{aligned}
$$

where $\lambda=\lambda_{\min }(Q) / \lambda_{\max }(\mathscr{H})-\mu_{1}, \varphi(t)=$ $\sup _{t \rightarrow \infty}\left\{\mu_{1}^{-1} \lambda_{\max }\left(\mathscr{P} \mathscr{B}^{T} \mathscr{H} \mathscr{B} \mathscr{P}\right)\|\widetilde{\mathbf{x}}\|^{2}\right\}$, and $\lambda_{\max }(\cdot)$ and $\lambda_{\text {min }}(\cdot)$ are the largest and smallest eigenvalues of a matrix. Hence, using global uniform ultimate boundedness (GUUB) stability [20], $V$ is exponential convergence, and the tracking error e can converge to a closed ball domain

$$
\Omega_{s}=\left\{\Theta \mid\|\oplus\|^{2} \leqslant \frac{\varphi(t)}{\lambda \cdot \lambda_{\min }(\mathscr{H})}\right\} .
$$

\section{Simulation Results}

In this section the intention is to evaluate the performance of the novel adaptive FTC. The evaluation is carried out on the 3-DOF model of UAV dynamics that can be found in [14]. The differential equations governing the point-mass UAV dynamics are given by

$$
\begin{gathered}
\dot{V}=g\left(\frac{T-D}{W}-\sin \gamma\right), \\
\dot{\gamma}=\frac{g}{V}(n \cos \mu-\cos \gamma), \\
\dot{\chi}=\frac{g n \sin \mu}{V \cos \gamma} .
\end{gathered}
$$

Flight trajectory can be generated by the following equations:

$$
\begin{gathered}
\dot{x}=V \cos (\gamma) \cos (\chi), \\
\dot{y}=V \cos (\gamma) \sin (\chi), \\
\dot{z}=V \sin (\gamma) .
\end{gathered}
$$

The state variables are airspeed $V$, flight path angle $\gamma$, and flight path heading angle $\chi$, and the control variables are 
TABLE 1: UAV model parameters.

\begin{tabular}{lc}
\hline Description & Value \\
\hline Density, $\rho$ & $1.2251 \mathrm{~kg} / \mathrm{m}^{3}$ \\
Weight, $W$ & $14,515 \mathrm{~kg}$ \\
Reference area, $S$ & $37.16 \mathrm{~m}^{2}$ \\
Maximum thrust, $T_{\max }$ & $113,868 \mathrm{~N}$ \\
Maximum lift coefficient, $C_{L_{\max }}$ & 2.0 \\
Maximum load factor, $n_{\max }$ & 7 \\
Induced drag coefficient, $k$ & 0.1 \\
Parasite drag coefficient, $C_{D_{0}}$ & 0.02 \\
\hline
\end{tabular}

thrust $T$, load factor $n$, and bank angle $\mu$. UAV position variables $x, y$, and $z$ are represented in the inertial frame. The drag force $D$ is represented by a simple drag polar model as

$$
D=0.5 \rho V^{2} S C_{D_{0}}+\frac{2 k n^{2} W^{2}}{\rho V^{2} S} .
$$

Detailed UAV model parameters are summarized in Table 1.

Let $x=[V, \gamma, \chi]^{T}, u_{c}=[T, n, \mu]^{T}$, and $y=x=h(x)$. The initial flight condition is a level flight with $V=300 \mathrm{~m} / \mathrm{s}$ at $z=3048 \mathrm{~m}$. To generate a differentiable command signal, reference command is transferred to the controller through the command filter $F=\omega_{n}^{2} /\left(s^{2}+2 \varsigma \omega_{n} s+\omega_{n}^{2}\right)$, where $\omega_{n}=$ $3 \mathrm{rad} / \mathrm{s}$ and $\varsigma=1$. In all simulations, it is assumed that the design objective is to assure that the forward velocity $V$ is regulated around the desired value $300 \mathrm{~m} / \mathrm{s}$, while the heading angle $\chi$ and flight path angle $\gamma$ follow 30 deg heading and 5 deg path angle commands as follows:

$$
\begin{gathered}
\chi_{d}= \begin{cases}0, & t<5, \\
6(t-5), & 5<t \leqslant 10 \\
30, & 10<t \leqslant 20 \\
30(5-0.2 t), & 20<t \leqslant 30 \\
-30, & 30<t \leqslant 40 \\
-30(9-0.2 t), & 40<t \leqslant 45, \\
0, & t>45\end{cases} \\
\gamma_{d}= \begin{cases}0, & t \leqslant 15 \\
0.5(t-15), & 15<t \leqslant 25 \\
5, & 25<t \leqslant 35 \\
0.5(45-t), & 35<t \leqslant 45 \\
0, & t>45\end{cases}
\end{gathered}
$$

It is assumed that the desired thrust $T$ and the applied thrust factor $T_{c}$ are related as $T_{c}=k_{n} T$, where $k_{n}$ denotes the thrust effectiveness coefficient such that $0<\sigma \leq k_{n} \leq 1$. In the nominal case $k_{n}=1$. The controller parameter is selected as $\mathscr{K}_{c}=\operatorname{diag}(1,1,1)$. The covariance of process and measurement is selected as $\mathbf{Q}=\operatorname{diag}\left(10^{-10}, 10^{-10}, 10^{-3}, 10^{-10}\right)$, $R=\operatorname{diag}\left(10^{-10}, 10^{-10}, 10^{-3}\right)$. The parameter of filter $(10)$ is chosen as $\zeta=50$.

The state responses of the UAV with the adaptive FTC and without FTC are shown in Figure 1. It is seen that the response is substantially improved compared to the case of
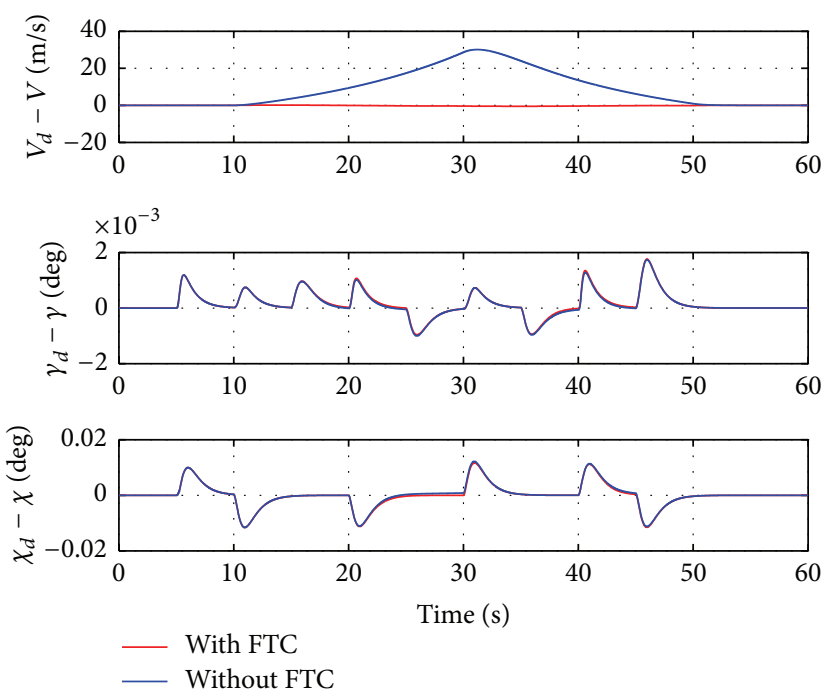

FIGURE 1: The system responses of the UAV.
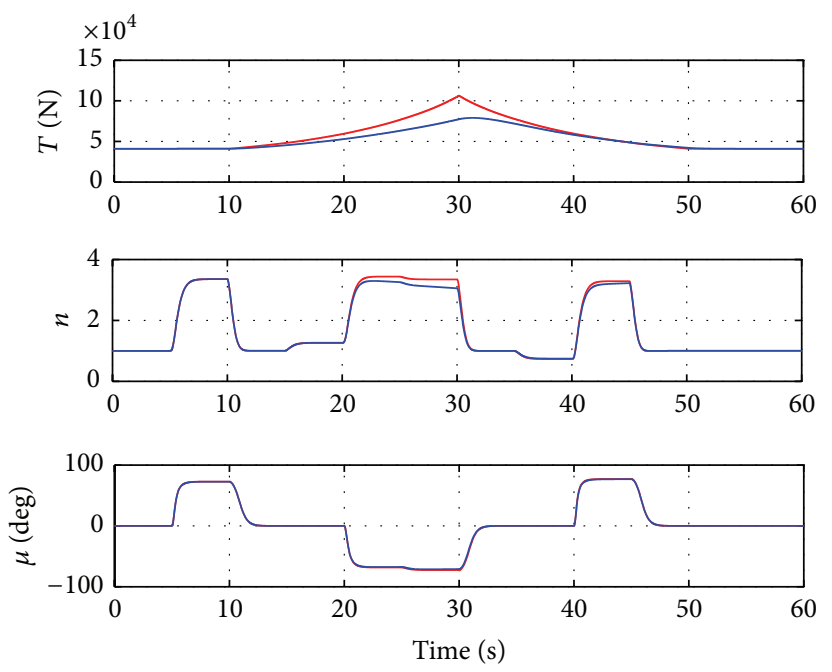

FIGURE 2: Control inputs.

without FTC. The control input can be seen in Figure 2. Figure 3 shows the 3D trajectories with FTC and without FTC, respectively. From Figure 4, it is seen that the estimate of thrust LOE factor $k_{n}$ converges to the true value.

\section{Conclusions}

This study deals with the fault-tolerant tracking control problem for nonaffine nonlinear systems. And a stability analysis was performed on the adaptive FTC law based on UKF. The proposed model approximation method is a solution that bridges the gap between affine and nonaffine control systems. The designed adaptive FTC strategy is applied to 3DOF simulation of a typical fighter aircraft, and simulation results are provided to demonstrate the effectiveness of the theoretic results obtained. Based upon the results presented in the paper, it is concluded that the fault-tolerant control 

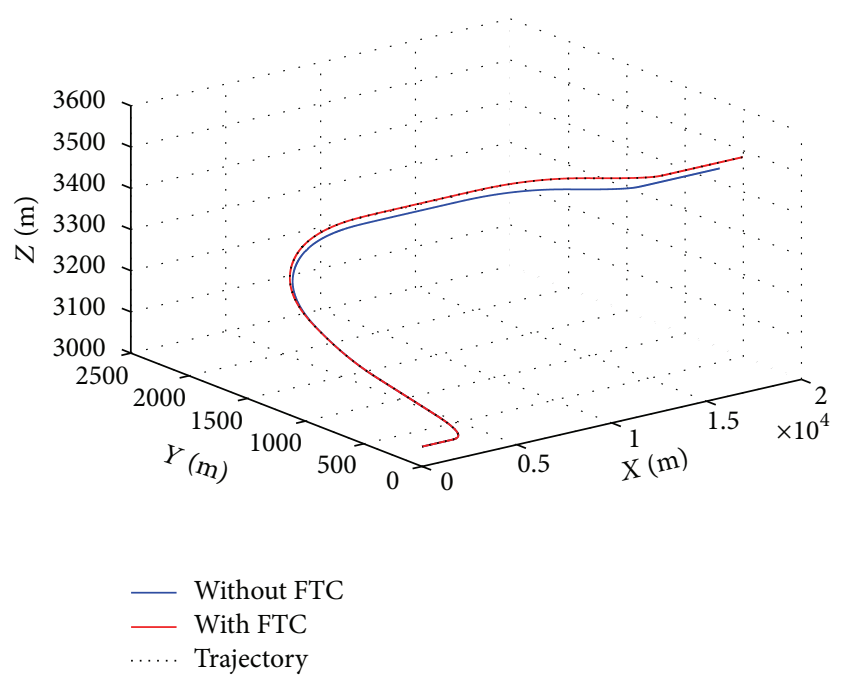

Figure 3: 3D trajectories.

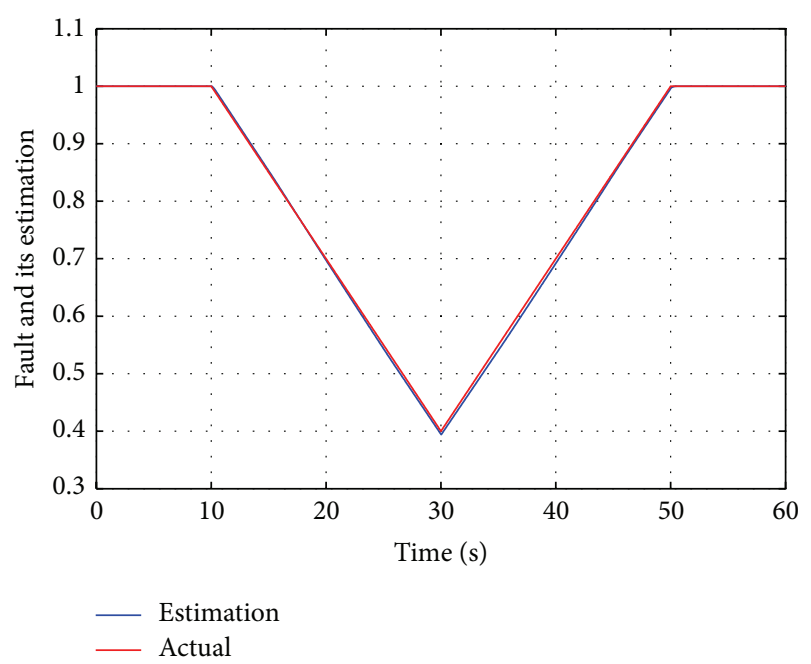

FIGURE 4: Response of the parameter estimates.

scheme successfully handles failures if actuators fail. The UKF-based controller was also able to track the kinematic states successfully during and after failures. After proposed nonaffine nonlinear tracking control, based on UKF, we further promote its conclusions to the fault-tolerant tracking control.

\section{Conflict of Interests}

The authors declare that there is no conflict of interests regarding the publication of this paper.

\section{Acknowledgments}

This work was partially supported by the National Natural Science Foundation of China (61374188) and the National Aerospace Science Foundation of China (2013ZC52033).

\section{References}

[1] Y. Zhang and J. Jiang, "Bibliographical review on reconfigurable fault-tolerant control systems," Annual Reviews in Control, vol. 32, no. 2, pp. 229-252, 2008.

[2] M. Blanke, M. Kinnaert, J. Lunze, and M. Staroswiecki, Diagnosis and Fault-Tolerant Control, Springer, Berlin, Germany, 2nd edition, 2006.

[3] H. Wu, "Reliable LQ fuzzy control for continuous-time nonlinear systems with actuator faults," IEEE Transactions on Systems, Man, and Cybernetics B: Cybernetics, vol. 34, no. 4, pp. 1743$1752,2004$.

[4] M. Mahmoud, "Stabilizing controllers for a class of discrete time fault-tolerant control systems," ICIC Express Letters, vol. 2, no. 3, pp. 213-218, 2008.

[5] Z. Gao, B. Jiang, P. Shi, and J. Liu, "Passive fault-tolerant control design for near-space hypersonic vehicle dynamical system," Circuits, Systems \& Signal Processing, vol. 31, no. 2, pp. 565-581, 2012.

[6] M. Liu, P. Shi, L. Zhang, and X. Zhao, "Fault-tolerant control for nonlinear Markovian jump systems via proportional and derivative sliding mode observer technique," IEEE Transactions on Circuits and Systems. I. Regular Papers, vol. 58, no. 11, pp. 2755-2764, 2011.

[7] Y. Song, X. Li, and W. Cai, "Adaptive and fault-tolerant reactive power compensation in power systems via multilevel STATCOMs," International Journal of Innovative Computing, Information and Control, vol. 9, no. 8, pp. 3403-3413, 2013.

[8] D. Xu, B. Jiang, H. Liu, and P. Shi, "Decentralized asymptotic fault tolerant control of near space vehicle with high order actuator dynamics," Journal of the Franklin Institute, vol. 350, no. 9, pp. 2519-2534, 2013.

[9] B. Jiang, M. Staroswiecki, and V. Cocquempot, "Fault accommodation for nonlinear dynamic systems," IEEE Transactions on Automatic Control, vol. 51, no. 9, pp. 1578-1583, 2006.

[10] Y. Xu, Y. Li, and S. Tong, "Fuzzy adaptive actuator failure compensation dynamic surface control of multiinput and multioutput nonlinear systems," International Journal of Innovative Computing, vol. 9, no. 12, pp. 4875-4888, 2013.

[11] H. Zhang, Y. Shi, and A. S. Mehr, "Robust static output feedback control and remote PID design for networked motor systems," IEEE Transactions on Industrial Electronics, vol. 58, no. 12, pp. 5396-5405, 2011.

[12] H. Zhang, Y. Shi, and M. Liu, " $H_{\infty}$ step tracking control for networked discrete-time nonlinear systems with integral and predictive actions," IEEE Transactions on Industrial Informatics, vol. 9, no. 1, pp. 337-345, 2013.

[13] H. Nijmeijer and A. Schaft, Nonlinear Dynamical Control Systems, Springer, New York, NY, USA, 1990.

[14] J. D. Bošković, L. Chen, and R. K. Mehra, "Adaptive control design for nonaffine models arising in flight control," Journal of Guidance, Control, and Dynamics, vol. 27, no. 2, pp. 209-217, 2004.

[15] D. Nesic, E. Skafidas, and I. M. Y. Mareels, "Minimum phase properties for input nonaffine nonlinear systems," IEEE Transactions on Automatic Control, vol. 44, no. 4, pp. 868-872, 1999.

[16] B. Jiang, Z. Gao, P. Shi, and Y. Xu, "Adaptive fault-tolerant tracking control of near-space vehicle using TakagiSugeno fuzzy models," IEEE Transactions on Fuzzy Systems, vol. 18, no. 5, pp. 1000-1007, 2010.

[17] C. Lin, C. Hsu, and R. Yeh, "Adaptive fuzzy sliding-mode control system design for brushless DC motors," International Journal 
of Innovative Computing, Information and Control, vol. 9, no. 3, pp. 1259-1270, 2013.

[18] X. Su, L. Wu, and P. Shi, "Sensor networks with random link failures: Distributed filtering for T-S fuzzy systems," IEEE Transactions on Industrial Informatics, vol. 9, no. 3, pp. 17391750, 2013.

[19] R. Kandepua, B. Foss, and L. Imslandb, "Applying the unscented Kalman filter for nonlinear state estimation," Journal of Process Control, vol. 18, no. 7-8, pp. 753-768, 2008.

[20] H. Khalil, Nonlinear Systems, Prentice Hall, New York, NY, USA, 3rd edition, 2002.

[21] H. Deng and H. Li, "A novel neural approximate inverse control for unknown nonlinear discrete dynamical systems," IEEE Transactions on Systems, Man, and Cybernetics B: Cybernetics, vol. 35, no. 1, pp. 115-123, 2005.

[22] D. Xu, B. Jiang, M. Qian, and J. Zhao, "Neural networks-based internal model optimal control for nonaffine nonlinear systems with input constraints," ICIC Express Letters B: Applications, vol. 3, no. 6, pp. 1341-1347, 2012.

[23] D. Xu, B. Jiang, and P. Shi, "Nonlinear actuator fault estimation observer: An inverse system approach via a T-S fuzzy model," International Journal of Applied Mathematics and Computer Science, vol. 22, no. 1, pp. 183-196, 2012.

[24] S. Julier, J. Uhlmann, and H. F. Durrant-Whyte, "A new method for the nonlinear transformation of means and covariances in filters and estimators," IEEE Transactions on Automatic Control, vol. 45 , no. 3 , pp. 477-482, 2000. 


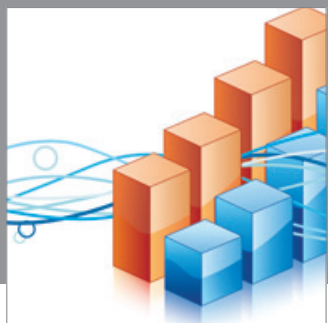

Advances in

Operations Research

mansans

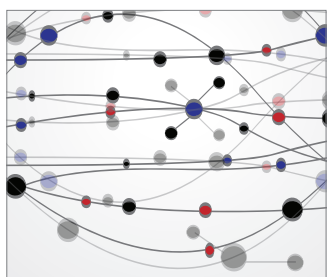

The Scientific World Journal
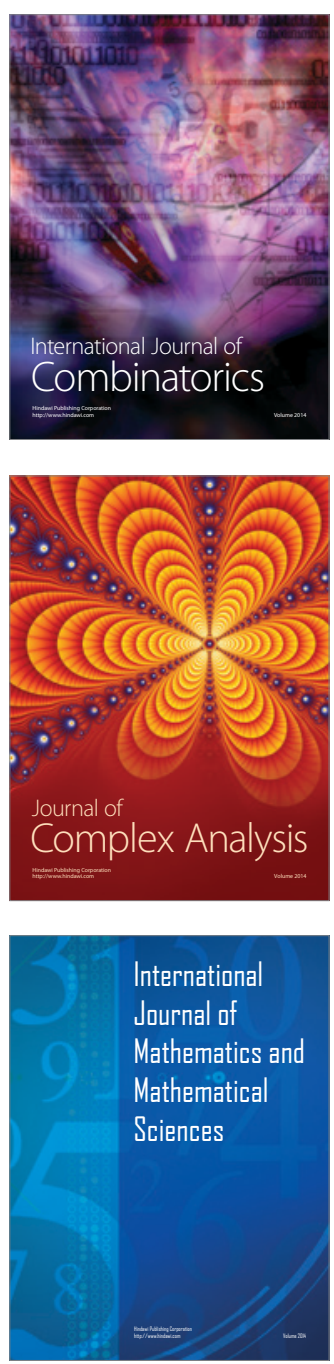
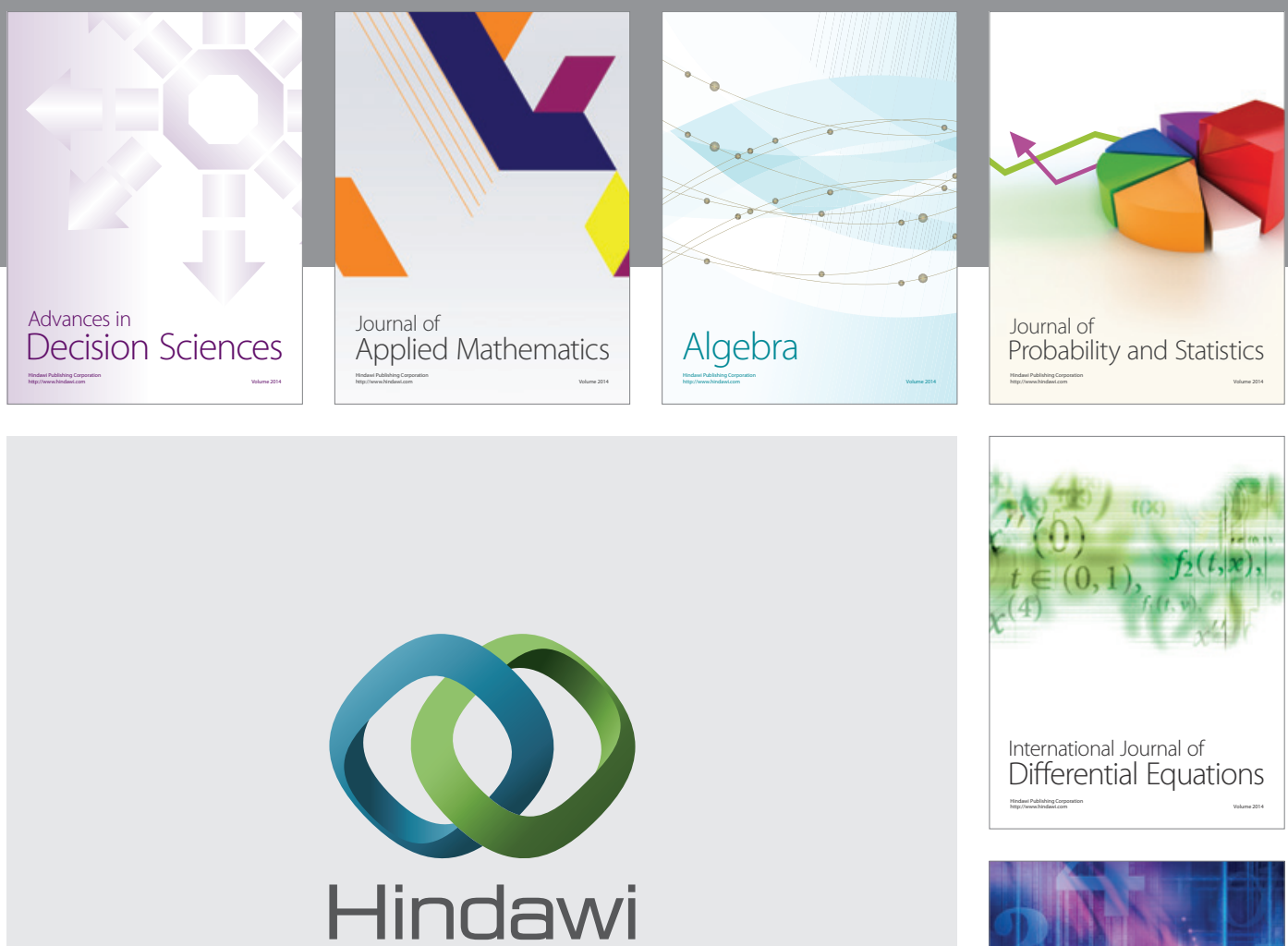

Submit your manuscripts at http://www.hindawi.com
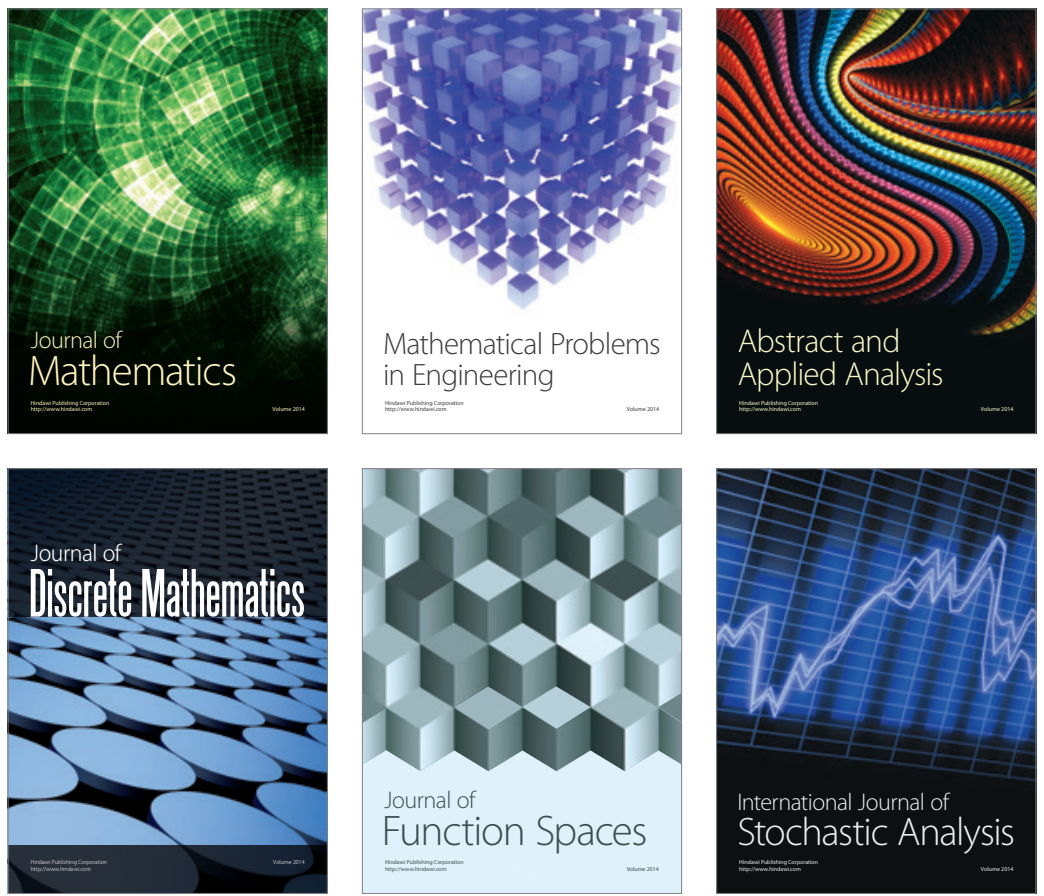

Journal of

Function Spaces

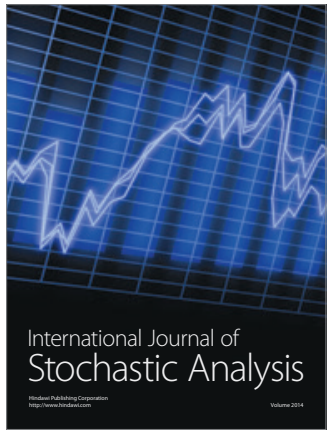

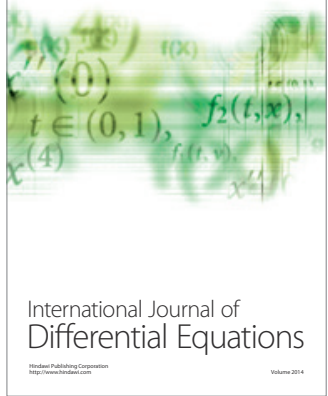
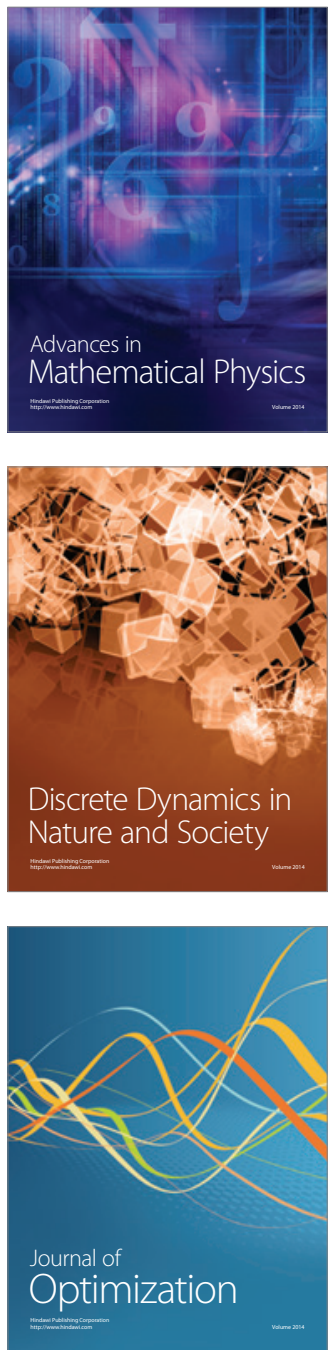